\title{
Interstitial lung diseases - a tertiary care center experience
}

\author{
Sreekala $\mathbf{C}^{1}$, Soofia $\mathbf{M}^{2}$, Sanjeev Nair ${ }^{3}, \mathrm{~K}_{\text {Anitha }}$ Kumari $^{4}$ \\ ${ }^{1}$ Dr. Sreekala C, ${ }^{2}$ Dr. M Soofiya, ${ }^{3}$ Dr. Sanjeev Nair, ${ }^{4}$ Dr. K Anitha Kumari, all authors are affiliated with \\ Department of Pulmonary Medicine, Medical College, Trivandrum, Kerala, India.
}

Address for Correspondence: Dr Sreekala C, Department of Pulmonary Medicine, Medical College, Trivandrum. C, Address: Navaneetham, APRA 307, AjanthaPulli Lane, Pettah PO, Chackai, Trivandrum. Email: drkalamurali@yahoo.co.in

\begin{abstract}
Introduction: Interstitial lung diseases are a group of diffuse parenchymal lung disorders associated with substantial morbidity and mortality and pose diagnostic and therapeutic challenges to the clinician. Diagnosis of ILD is by multidisciplinary discussion (MDD) between clinician, radiologist and pathologist. The incidence and prevalence of ILDs in India are unknown. Prospective disease registries can provide better estimates of incidence and prevalence as well as insights to etiology, associated risks, natural history, and outcomes of a disease. This was a prospective study undertaken to analyse the spectrum of ILD encountered, demographic profile, clinical and radiological characteristics of the patients with interstitial lung disease in a tertiary care setting. Materials and Methods: A prospective study over a period of two years conducted at Medical College,Thiruvananthapuram. 164 consecutive patients with multidisciplinary diagnosis of interstitial lung disease were included in the study. Detailed history, spirometry, SPO2, DLCO, HRCT, 6 minute walk test, ECG, 2D ECHO were done and recorded in duly filled proforma.ANA profile, serum angiotensin converting enzyme,serum calcium, 24 hour urinary calcium were estimated wherever it was indicated. Diagnosis of IPF was done after excluding the known causes and the HRCT findings suggestive of UIP pattern based on the current guidelines of the American Thoracic Society/European Respiratory Society 2013. Statitical Analysis. Data were entered in Microsoft Excel and analyzed using Epi Info version 7. For descriptive statistics, quantitative variables were described by mean and standard deviation. Qualitative variables were described by percentage distribution. For inferential statistics between groups, comparison of qualitative variables were analysed by chi-square test and quantitative variables were compared by student $t$ test. $\mathrm{P}$ value of less than 0.05 was considered as level of significance. Result: 164 consecutive ILD patients were included in the study.Of this $26.26 \%$ patients were IPF and $73.74 \%$ were non-IPF. CTD-ILD was the most common (64\%). Mean age of the study population was $54.03 \pm 11.08$ years. The mean age was higher among IPF patients $(61.41 \pm 7.7)$ compared to non-IPF ILD patients $(51.4 \pm 10.9) .65 .8 \%$ of study populations were were females. Among IPF patients, $65.1 \%$ were males (95\%CI: 49.07\%, 78.99\%) compared to $23.2 \%$ (95\% CI: $15.96 \%, 31.68 \%$ ) males in non- IPF group $(\mathrm{p}<.001)$. The proportion of ever smokers was higher among IPF patients $(62.7 \%), 95 \%$ CI: 46.7\%, $77.02 \%)$ compared to non IPF group $(15.7 \%)$, 95\% CI: 9.7\%, 23.43\%) (p<.001). IPF patients had lower mean FVC \% compared to non- IPF ILDs. $(48.1 \pm 16.2$ vs $51.2 \pm 23.8)(\mathrm{p}<.001)$. The mean DLCO was lower among IPF patients $(42.6 \pm 9.92$ vs $53.9 \pm 15.22)$ compared to non-IPF group at presentation $(\mathrm{p}<.002)$. The mean 6 MWD was lower among IPF patients $(209.6 \pm 96.30$ meters compared to non-IPF $(288.6 \pm 101.2)$ meters $(\mathrm{P}<.001)$. IPF patients had lower mean SpO2 (90.13 \pm 3.5$)$ compared to non-IPF group $(93.82 \pm 2.86) .100 \%$ of IPF patients had UIP pattern in HRCT where as, $9.92 \%$, of patients (95\% CI: $5.2 \%, 16.6 \%)$ among non-IPF group had UIP pattern in HRCT $(\mathrm{p}<.001)$. In IPF patients, the proportion of patients with pulmonary HTN was higher $(53.49 \%$, 95\% CI: $37.65 \%, 68.82 \%)$ compared to non-IPF $(21.49 \%, 95 \%$ CI: 14.5\%, 29.8\%). GERD symptoms were more common among IPF patients (74.4\%) compared to $67.7 \%$ among non IPF, which was not statistically significant. ( $\mathrm{p}$ 0.41).Conclusion. Out of 164 ILD patients, 26.2\% were IPF. Among non -IPF group, CTD-ILD was the commonest. The mean age was higher among IPF patients, compared to non-IPF ( $\mathrm{p}$ value $=0.001$.)IPF was more common in males, where as females predominate in Non-IPF group $(\mathrm{p}<0.001)$ The proportion of ever smokers was higher among IPF patients (62.7\%). FVC\%, DLCO \%, 6MWD, SPO2 were lower in IPF compared to non $-\mathrm{IPF}$, at the time of presentation $(\mathrm{p}<0.001)$. HRCT showed UIP pattern in $100 \%$ of IPF. Proportion of
\end{abstract}


patients with pulmonary HTN was higher in IPF (53.49\%) compared to non-IPF (21.49\%). GERD symptoms were present in $69.5 \%$ of study population.

Keywords: Idiopathic pulmonary fibrosis (IPF), Non idiopathic pulmonary fibrosis (Non-IPF), Connective tissue - ILD (CTD-ILD)

\section{Introduction}

Interstitial lung diseases are a group of diffuse parenchymal lung disorders associated with substantial morbidity and mortality[1]. Interstitial lung disease (ILD), also referred to as diffuse parenchymal lung disease, is a heterogeneous group of lung diseases Interstitial lung diseases (ILDs) are a heterogeneous group of acute and chronic bilateral lung diseases of known and unknown causes and pose diagnostic and therapeutic challenges to the clinician. Diagnosis of ILD is by multidisciplinary discussion (MDD) between clinician, radiologist and pathologist[1]. Knowledge achieved in recent years has resulted in the new classification of idiopathic interstitial pneumonias.

International Multidisciplinary Consensus Classification of Idiopathic Interstitial Pneumonias by American Thoracic Society / European Respiratory Society 2013 is useful in classifying ILD, according to which there are three groups: major, rare and unclassified [2] Idiopathic pulmonary fibrosis is the most lethal amongst the interstitial lung diseases and presents high heterogeneity in clinical behaviour The understanding of the distinct appearance of the diffuse parenchymal lung diseases in high resolution computed tomography (HRCT) has greatly reduced the need for biopsy of the condition.

The incidence and prevalence of ILDs in India are unknown [3]. Prospective disease registries can provide better estimates of incidence and prevalence as well as insights to etiology, associated risks, natural history, and outcomes of a disease [3]. This was a prospective study undertaken to analyse the spectrum of ILD encountered, demographic profile, clinical and radiological characteristics of the patients with interstitial lung disease in a tertiary care setting.

Manuscript received: $28^{\text {th }}$ May 2017

Reviewed: $8^{\text {th }}$ June 2017

Author Corrected: $18^{\text {th }}$ June 2017

Accepted for Publication: $25^{\text {th }}$ June 2017

\section{Materials and Methods}

We conducted a prospective study over a period of two years at Medical College, Thiruvananthapuram. 164 consecutive patients with multidisciplinary diagnosis of interstitial lung disease attending Medical college, Thiruvananthapuram were included in the study. Patients with ILD secondary to malignancy were excluded from the study.

The data on age, sex, occupation, presenting complaints, smoking status, environmental exposures, history of anti-tuberculous treatment, connective tissue diseases, family history of ILD and physical examination findings were recorded using duly filled proforma. All the patients with suspected ILD were subjected to HRCT thorax. Contrast CT Thorax was done in patients suspected to have mediastinal lymphadenopathy. Spirometry and diffusion capacity for carbon monoxide (DLCO), SPO2 measurement were done for all patients. Six-minute walk test (6MWT) was performed whenever patient's condition permitted for the same. Baseline 2D echocardiogram was done in all the patients.

The Antinuclear antibody (ANA) profile, Rheumatoid factor (RF), Anti- cyclic citrullinated peptide (Anti-CCP), Antinuclear cytoplasmic antibody (ANCA) and serum Angiotensin converting enzyme, serum calcium, 24 hour urinary calcium were estimated wherever it was indicated. Diagnosis of IPF was done after excluding the known causes and the HRCT findings suggestive of UIP pattern based on the current guidelines of the American Thoracic Society/European Respiratory Society 2013.

Statistical Analysis: Data entered in Microsoft Excel and analyzed using Epi Info version 7. For descriptive statistics, quantitative variables measured by mean and standard deviation. Qualitative variables described by percentage distribution. For inferential statistics between groups, comparison of qualitative variables were 
analysed by chi-square test and quantitative variables were compared by student $\mathrm{t}$ test. $\mathrm{P}$ value of less than 0.05 was consider as level of significance.

\section{Results}

164 patients with ILD, diagnosed by Multi-disciplinary discussion were recruited. Out of this 43 patients (26.2\%); 95\% CI: $19.67 \%, 33.65 \%)$ were idiopathic pulmonary fibrosis (IPF) and $121(73.7 \%)$; $95 \%$ CI: $66.35 \%, 80.35 \%$ ) were non - IPF.

Fig.-1: Proportion of IPF among Interstitial lung diseases

Table-1: Proportion of various non-IPF ILDs.

\begin{tabular}{|c|c|c|c|c|c|}
\hline & & & & \multicolumn{2}{|c|}{$95 \% \mathrm{CI}$} \\
\hline & $\begin{array}{c}\text { Number of } \\
\text { patients }\end{array}$ & $\begin{array}{c}\text { Proportion of non-IPF } \\
\text { ILD }\end{array}$ & $\begin{array}{c}\text { As a proportion of total } \\
\text { ILD }\end{array}$ & Lower & Higher \\
\hline CTD ILD & 75 & $64 \%$ & $45.73 \%$ & $37.94 \%$ & $53.68 \%$ \\
\hline Idiopathic NSIP & 16 & $14 \%$ & $10.37 \%$ & $6.16 \%$ & $16.08 \%$ \\
\hline Sarcoidosis & 6 & $5 \%$ & $3.66 \%$ & $1.35 \%$ & $7.79 \%$ \\
\hline HSP & 12 & $10 \%$ & $7.93 \%$ & $4.29 \%$ & $13.17 \%$ \\
\hline COP & 3 & $2 \%$ & $1.83 \%$ & $0.38 \%$ & $5.25 \%$ \\
\hline DIP & 1 & $1 \%$ & $0.61 \%$ & $0.02 \%$ & $3.35 \%$ \\
\hline $\mathrm{LCH}$ & 1 & $1 \%$ & $0.61 \%$ & $0.02 \%$ & $3.35 \%$ \\
\hline IPH & 1 & $1 \%$ & $0.61 \%$ & $0.02 \%$ & $3.35 \%$ \\
\hline Early ILD & 2 & $2 \%$ & $1.83 \%$ & $0.38 \%$ & $5.25 \%$ \\
\hline
\end{tabular}

Among non-IPF ILDs, Connective tissue disease related ILD (CTD-ILD) was the most common (64\%), followed by idiopathic NSIP (14\%)

\section{Demographic Features}

AGE

Table-2: Distribution of age in ILDs.

\begin{tabular}{|c|c|c|c|c|c|}
\hline & Median & Minimum & Maximum & \multicolumn{2}{|c|}{ Inter quartile range } \\
\hline & & & & $25^{\text {th }}$ percentile & 75 th percentile \\
\hline Non IPF & 53 & 25 & 75 & 45 & 59 \\
\hline IPF & 62 & 43 & 76 & 58 & 68 \\
\hline
\end{tabular}

Mean age of the study population was $54.03 \pm 11.08$ years. The mean age was higher among IPF patients $(61.41 \pm 7.7)$ compared to non-IPF ILD patients $(51.4 \pm 10.9)$. The median age of the IPF group was 62 years and that of non -IPF group was 53 years. The difference in the median age in both groups was statistically significant ( $\mathrm{p}$ value $=0.001$.

\section{SEX}

Out of the 164 ILD patients, $65.8 \%$ were females (95\%CI 58.05\%, 73.07\%) and $34.1 \%$ were males (95\% CI: $26.93 \%, 41.95 \%)$. Among IPF patients, $65.1 \%$ were males $(95 \% \mathrm{CI}$ : $49.07 \%, 78.99 \%)$ compared to $23.2 \%(95 \%$ CI: $15.96 \%, 31.68 \%$ ) males in non- IPF group.

\section{Smoking status}

Among the study population, $28.05 \%$ of patients (95\% CI $21.3 \%, 35.5 \%$ ) were ever smokers. 
The proportion of ever smokers was higher among IPF patients (62.7\%), 95\% CI: 46.7\%, 77.02\%) compared to non IPF group (15.7\%), 95\% CI: 9.7\%, 23.43\%). The difference in the proportion of smokers was statistically significant. (RR- 2.09, 95\% CI: 1.4, 2.97) p value $<0.001$

Forced vital capacity (FVC\% predicted)

Table 3: distribution of FVC\% among ILDs

\begin{tabular}{|c|c|c|c|c|c|}
\hline & Median & Minimum & Maximum & \multicolumn{2}{|c|}{ Inter quartile range } \\
\hline & & & & $25^{\text {th }}$ percentile & 75 th percentile \\
\hline Non IPF & 49 & 17 & 103 & 40 & 64 \\
\hline IPF & 41 & 15 & 128 & 33 & 64 \\
\hline
\end{tabular}

- $\quad$ The mean \% predicted FVC of the study population was 50.4 \pm 18.5 .

- IPF patients had lower mean FVC \% compared to non- IPF ILDs. (48.1 \pm 16.2 vs 51.2 \pm 23.8 ).

- The difference between median FVC of two groups was statistically significant $\mathrm{p}$ value $=0.001$

Diffusion lung capacity for carbon monoxide (DLCO\%predicted.)

Table 4: distribution of DLCO\% among ILDs.

\begin{tabular}{|c|c|c|c|c|c|}
\hline & Median & Minimum & Maximum & \multicolumn{2}{|c|}{ Inter quartile range } \\
\hline & & & & $25^{\text {th }}$ percentile & 75 th percentile \\
\hline Non IPF & 53 & 20 & 111 & 47 & 61 \\
\hline IPF & 40 & 18 & 62 & 35 & 50 \\
\hline
\end{tabular}

- The mean DLCO \% of the population was $50.4 \pm 14.7$.

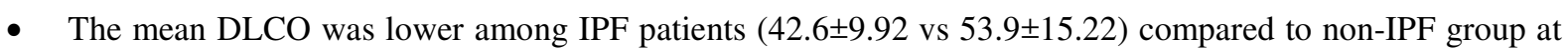
presentation. The difference in median DLCO was statistically significant. $p$ value $=0.002$

\section{Minute walk distance (6MWD)}

- The mean 6MWD of the study population was $267.2 \pm 105.6$ meters.

- The mean 6MWD was lower among IPF patients (209.6 \pm 96.30 meters compared to non-IPF $(288.6 \pm 101.2)$ meters.

- The difference of mean 6MWD in two groups was statistically significant. $\mathrm{p}$ value $<0.001$

\section{$\mathrm{O}_{2}$ Saturation (SpO2)}

- The mean saturation of the study population was $92.8 \pm 3.4 \%$.

- IPF patients had lower mean SpO2 (90.13 \pm 3.5$)$ compared to non-IPF group(93.82 \pm 2.86$)$.

- The difference in mean $\mathrm{SpO} 2$ was statistically significant in both groups. $\mathrm{p}$ value $<0.001$

\section{HRCT - UIP Pattern}

UIP pattern in HRCT was present in 33.5\% patients (95\% CI: 26.3, 41.2). 100\% of IPF patients had UIP pattern in HRCT where as, $9.92 \%$, of patients (95\% CI: 5.2\%, 16.6\%) among non-IPF group had UIP pattern in HRCT. The difference in the proportion of UIP pattern in HRCT in two groups was statistically significant. (RR- 4.5, $95 \%$ CI: $2.7,7.5)$. p value $<0.001$ 
GERD symptoms were present in $69.5 \%$ of study population. (95\% CI: 61.8, 76.4), which was more common among IPF patients $(74.4 \%)$ compared to $67.7 \%$ among non IPF.

The difference in proportion of GERD symptoms in two groups was not statistically significant. (RR $1.08,95 \%$ CI: $0.8,1.3 \mathrm{p}$ value 0.41$)$.

Pulmonary hypertension- In the study $29.8 \%$ of patients ( $95 \%$ CI: $22.9,37.5$ ) had pulmonary hypertension. In IPF patients, the proportion of patients with pulmonary HTN was higher $(53.49 \%, 95 \%$ CI: 37.65\%, 68.82\%) compared to non-IPF $(21.49 \%, 95 \% \mathrm{CI}: 14.5 \%, 29.8 \%)$.

The difference in proportion of patients with pulmonary hypertension in both groups was statistically significant. (RR-1.5, 95\% CI: 1.18, 2.05). p value $<0.001$ Top of Form

\section{Discussion}

164 patients with diagnosis of ILD were included in our study, out of which 43 patients $(26.2 \%)$ IPF and121 $(73.7 \%)$ were non-IPF similar to that reported by Sharma et al[11]. But Kumar Adesh et al reported a prevalence of $40.5 \%$ for IPF [4]. Somenath Kundu et al reported that IPF was found to be the most common ILD (38.04\%) followed by CTD - ILD (31.5\%)[6].

In our study Connective tissue disease related ILD (CTD-ILD) was the most common (64\%) non- IPF ILD followed by idiopathic NSIP (14\%) But in a study published by Singh et al, Hypersensitivity pneumonitis (HP) was diagnosed in $47.3 \%$ connective tissue disease-associated ILD in $13.9 \%$ and IPF in $13.7 \%[3]$.

Mean age of the study population was $54.03 \pm$ 11.08 years. According to previous study, mean age at presentation was 45.24 years[4].

The mean age was higher among IPF patients (61.41 \pm 7.7$)$ compared to non-IPF ILD patients (51.4 \pm 10.9$)$. Kumar Adesh et al reported a mean age of 54.83 years in IPF, 44.60 years in HSP, 43.60 years in NSIP and 41.56 years in Sarcoidosis [4].

The CTD-DPLD group had a lower mean age (39.5 \pm 1.86 vs $56.9 \pm 1.12$ years), a longer duration of symptoms [6].

$65.8 \%$ were females and $34.1 \%$ were males in our study correlating with the result of the study by Kumar Adesh et al.[4] Among IPF patients, 65.1\% were males compared to $23.2 \%$ males in non- IPF group in our study, similar to result of previous study[4].
In this study $28.05 \%$ of patients were ever smokers., which was lower than earlier reports where $(51.7 \%)$ were smoker[4].

The proportion of ever smokers was higher among IPF patients $(62.7 \%)$, compared to non IPF group $(15.7 \%)$ in our study, earlier study showed that smoking was present in $78.70 \%$ cases of IPF[4].

The mean $\%$ predicted FVC of the study population was $50.4 \pm 18.5$. The mean FVC was $58.3 \%$ of predicted in previous study [4]. IPF patients had lower mean FVC\% compared to non- IPF ILDs. $(48.1 \pm 16.2$ vs $51.2 \pm 23.8)$ correlating with earlier study result[5]. According to Raj Kumar et al Mean of predicted total lung capacity (TLC) was 64.3\%, the lowest being in the IPF group (58.88\%).[12]

The mean DLCO $\%$ of the population was $50.4 \pm 14.7$ in our study, similar to earlier result by Singh et al where DLCO was $45.56 \%$ of predicted [4].

The mean DLCO was lower among IPF patients (42.6 \pm 9.92 vs $53.9 \pm 15.22)$ compared to non-IPF group at presentation., Kumar Ret al reported,that mean of predicted DLCO was $50.56 \%$, the lowest being in the IPF group (42.75\%)[5].

Study by to Raj Kumar et al showed that Mean of predicted DLCO was $50.56 \%$, the lowest being in the IPF group (42.75\%).[12].

The mean 6MWD was lower among IPF patients (209.6 \pm 96.30 meters compared to non-IPF (288.6 \pm 101.2$)$ meters, as reported in previous study $[5,7]$. 
The mean saturation of the study population was $92.8 \pm 3.4 \%$. IPF patients had lower mean $\mathrm{SpO} 2$ $(90.13 \pm 3.5)$ compared to non-IPF group (93.82 \pm 2.86$)$, correlating with earlier result $[5,7]$.

UIP pattern in HRCT was present in $33.5 \%$ study population $100 \%$ of IPF patients had UIP pattern in HRCT where as only $9.92 \%$, of patients among non-IPF group had UIP pattern in HRCT Somenath Kundureported that all cases of IPF $(100 \%)$ showed a classical UIP pattern on the HRCT thorax [6]. In the study by Khantal et al typical radiographic findings were observed on HRCT in $90 \%$ patients [13]

GERD symptoms were more common among IPF patients $(74.4 \%)$ compared to $67.7 \%$ among non IPF, in our study, a finding that was not statistically significant. $p$ value 0.41 ).

In the study $29.8 \%$ of patients had pulmonary hypertension based on 2D ECHO. In IPF patients, the proportion of patients with pulmonary HTN was higher $(53.49 \%)$, compared to non-IPF $(21.49 \%)$. The prevalence of $\mathrm{PH}$ in the setting of IPF has not been well described in the literature, with a reported occurrence from $32 \%$ to $85 \%$ [8].

\section{Conclusion}

The incidence and prevalence of ILDs in India are unknown. Prospective disease registries can provide better estimates of incidence and prevalence as well as insights to etiology, associated risks, natural history, and outcomes of a disease. This prospective study analyzed the spectrum of ILD encountered, demographic profile, clinical and radiological characteristics of the patients with interstitial lung disease in a tertiary care setting.

\section{Funding: Nil, Conflict of interest: None Permission of IRB: Not required}

\section{References}

1.KaterinaM. Antoniou, George,A.Margaritopoulos Sara Tomassetti. Interstitial lung disease. European Respiratory Review 2014 23: 40-54.

2. William D. Travis, Ulrich Costabel, David M. Hansell, Talmadge E. King, Jr., David A. Lynch, Andrew G. An Official American Thoracic Society/European Respiratory Society Statement:
Update of the International Multidisciplinary Classification of the Idiopathic Interstitial Pneumonias 2013

3. Ganesh Raghu and Sanjeev Mehta. Interstitial lung disease (ILD) in India: Insights and lessons from the prospective, landmark ILD-India registry. Lung India. 2016 Nov-Dec; 33(6): 589-591.

4. Kumar Adesh, Yadav Prashant, Gupta K. Ashish, Gautam K. Aditya, Kumar Anand. Profile of interistial lung diseases at tertiary care centre of northern India. European journal of pharmaceuticaland medical research, 2016, 3 (8), 368-374.

5. Kumar R, Gupta N, Goel N. Spectrum of interstitial lung disease at a tertiary care centre in India. Pneumonol Alergol Pol. 2014;82(3):218-26. doi: 10.5603/PiAP.2014.0029.

6. Somenath Kundu, Subhra Mitra, Joydeep Ganguly, Subhasis Mukherjee, Souvik Ray Ritabrata Mitra. Spectrum of diffuse parenchymal lung diseases with special reference to idiopathic pulmonary fibrosis and connective tissue disease: An eastern India experience. Lung India 2014;31: 354-60.

7. Garin MC, Highland KB, Silver RM, Strange C. Limitations to the 6-minute walk test in interstitial lung disease and pulmonary hypertension in scleroderma. J Rheumatol. 2009 Feb; 36 (2): 330-6. doi: 10.3899 /jrheum.080447. Epub 2009 Jan 22.

8. Smith JS, Gorbett D, Mueller J, Perez R, Daniels CJ. Pulmonary hypertension and idiopathic pulmonary fibrosis: a dastardly duo. Am J Med Sci. 2013;346(3):221-5.

9. Sheetu Singh Bridget F. Collins, Bharat B. Sharma Jyotsna M. Joshi. Interstitial Lung Disease in India. Results of a Prospective Registry. American Journal of Respiratory and Critical Care Medicine Vol. 195, No. 6, 2017

10. The ILD India Registry: A Novel Tool for Epidemiological Surveillance of Interstitial Lung Disease in India. Indian $\mathrm{J}$ Chest Dis Allied Sci 2013;55:197-199] 


\section{Original Research Article}

11. Singh V, Sharma BB. Laying the ground for research of interstitial lung disease in our country: ILD India registry. Lung India. 2014 Oct;31(4): 320-2. doi: 10.4103/0970-2113.142091.

12. Kumar R, Gupta N, Goel N. Spectrum of interstitial lung disease at a tertiary care centre in
India. Pneumonol Alergol Pol. 2014;82(3):218-26. doi: 10.5603/PiAP.2014.0029.

13. Khantal N, Dubey A. Profile of Idiopathic Pulmonary Fibrosis cases at a tertiary care Institute. Int J Med ResRev 2015; 3(7):717-720.doi:10. 17511/ijmrr.2015.i7.135.

\section{How to cite this article?}

Sreekala C, Soofia M, Sanjeev Nair, K Anitha Kumari. Interstitial lung diseases - a tertiary care center experience. Int J Med Res Rev 2017;5(07):657-663. doi:10.17511/ijmrr. 2017.i07.03. 